Instituto Internacional de Investigación y Desarrollo Tecnológico Educativo INDTEC, C.A.

DOI: https://doi.org/10.29394/Scientific.issn.2542-2987.2020.5.18.1.24-45

OAI-PMH: http://www.indteca.com/ojs/index.php/Revista Scientific/oai

Artículo Original / Original Article

\title{
Gestión de la Administración Escolar en el Desarrollo de Actividades Académicas: Mirada en Tiempos de Pandemia
}
Autoras: Amely Dolibeth Vivas Escalante Universidad Miguel de Cervantes, UMC amelydvivase@gmail.com
Santiago Chile http://orcid.org/0000-0002-5791-8619

Marlenis Marisol Martínez Fuentes Universidad Miguel de Cervantes, UMC marlenism3@gmail.com

Santiago, Chile https://orcid.org/0000-0002-5926-599X

Doris Josefina Solís Mejías Universidad Miguel de Cervantes, UMC dorisolis@hotmail.com

Santiago Chile http://orcid.org/0000-0001-6413-1075

\section{Resumen}

El objetivo de la presente investigación fue analizar los procesos administrativos de la gerencia educativa en el desarrollo de las actividades académicas del Liceo Técnico Amelia Courbis, ubicado en Talca, Chile. El aspecto metodológico se enmarcó dentro del tipo de investigación descriptiva, la muestra estuvo constituida por 25 directivos-docentes que conforman el $30 \%$ de la población a quienes se les aplicó un cuestionario con la escala de Likert, los datos obtenidos fueron procesados estadísticamente teniendo una confiablidad de 0.93 en la formula alfa de Cronbach, se observó que los procesos administrativos de control y organización no se están llevando a cabo rigurosamente afectando la estabilidad institucional, la gerencia educativa se encuentra enmarcada en un estilo gerencial autocrático evitando la participación activa del personal en el proceso educativo, asimismo los docentes consideran que su director carece de preparación necesaria para llevar a cabo la gerencia de la institución, debido a que no cumple con las características propias para el cargo.

Palabras clave: gestión; administración; actividades.

\section{Cómo citar este artículo:}

Vivas, A., Martínez, M., \& Solís, D. (2020). Gestión de la Administración Escolar en el Desarrollo de Actividades Académicas: Mirada en Tiempos de Pandemia. Revista Scientific, 5(18), 24-45, eISSN: 2542-2987. Recuperado de: https://doi.org/10.29394/Scientific.issn.2542-2987.2020.5.18.1.24$\underline{45}$

Fecha de Recepción: 19-06-2020
Fecha de Aceptación: 23-09-2020
Fecha de Publicación: 05-11-2020 
OAI-PMH: http://www.indteca.com/ojs/index.php/Revista_Scientific/oai

Artículo Original / Original Article

\title{
Management of School Administration in the Development of Academic Activities: A Look in Times of Pandemic
}

\begin{abstract}
The objective of this research was to analyze the administrative processes of educational management in the development of academic activities at the Amelia Courbis Technical High School, located in Talca, Chile. The methodological aspect was framed within the type of descriptive research, the sample consisted of 25 directors-teachers who make up $30 \%$ of the population to whom a questionnaire with the Likert scale was applied, the data obtained were statistically processed having a reliability of 0.93 in Cronbach's alpha formula, it was observed that the administrative processes of control and organization are not being carried out rigorously affecting institutional stability, educational management is framed in an autocratic managerial style avoiding the active participation of personnel in the educational process, also the teachers consider that their director lacks the necessary preparation to carry out the management of the institution, because he does not meet the characteristics of the position.
\end{abstract}

Keywords: management; administration; activities.

\footnotetext{
How to cite this article:

Vivas, A., Martínez, M., \& Solís, D. (2020). Management of School Administration in the Development of Academic Activities: A Look in Times of Pandemic. Revista Scientific, 5(18), 2445, e-ISSN: 2542-2987. Recovered from: https://doi.org/10.29394/Scientific.issn.25422987.2020.5.18.1.24-45
}

Date Received: 19-06-2020
Date Acceptance: 23-09-2020
Date Publication: 05-11-2020 
OAI-PMH: http://www.indteca.com/ojs/index.php/Revista_Scientific/oai

Artículo Original / Original Article

\section{Introducción}

La educación latinoamericana, para Muñoz y Villa (2017): ha modificado sus procesos de reformas, originando cambios en los estilos de desarrollo económico, social y político con el que se enfrentan a nuevas estructuras, espacios donde el gerente dirige los procesos de cambio logrando los objetivos pautados en las diferentes esferas del quehacer del hombre. Por lo tanto, la educación en el mundo está experimentando cambios y transformaciones continuas que indicen directamente en todas las esferas de la sociedad a fin de satisfacer los intereses que conllevan a los cambios.

De acuerdo a lo planteado, la administración en la praxis educativa es esencial porque se planifica, organiza, ejecuta y controla las actividades y demás recursos que complementan el trabajo pedagógico. De acuerdo con Miranda (2016): el proceso administrativo aborda la factibilidad del tiempo, estrategias, recursos, condiciones a fin de dar a conocer el trabajo, de acuerdo a los objetivos y metas planteadas.

Es de resaltar que el encuentro educativo implica actividades pedagógicas-administrativas, las cuales son llevadas a cabo por el personal, orientados por la dirección, ente rector que gerencia y conduce el liderazgo dentro del entorno educativo. Mencionando a Fierro-Evans y Carbajal-Padilla (2019): el directivo, como gerente del personal, los recursos debe inducir a equipos de trabajo colaborativo y logrando la mediación en la resolución de los conflictos y proporcionando un clima de convivencia apropiado.

Las instituciones escolares se han visto afectadas por transformaciones políticas, ambientales y sociales que han incidido en el docente en las actividades que efectúa en su labor diaria. Según Chávez (2017): los directivos y educadores tienen un papel importante en el progreso de un país y, en la praxis se realizan muchas acciones, tales como: académicas, administrativas, comunitarias, sociales, culturales, artísticas, entre otras.

En Chile no se escapa la realidad a la que se viene haciendo referencia, 
dado que con el confinamiento de los directivos producto de la pandemia COVID-19, han tenido que implementar nuevas modalidades en la gestión de la administración escolar en el desarrollo de las actividades académicas a través de la educación remota o virtual, sin contar con las condiciones de trabajo y conectividad adecuada, sin una preparación en el uso de herramientas tecnológicas y con horarios de trabajo más extensos de los que venían realizando.

De allí que, en el Liceo Técnico Amelia Courbis, ubicado en la provincia Talca, por medio de entrevistas no formales realizada a algunos directivos expresaron que se está incumplimiento con los recaudos por parte de los docentes, ausencia de corresponsabilidad de parte de los apoderados, falta de tolerancia, comunicación entre estudiantes-docentes y poco manejo de la tecnología para dar respuestas inmediatas a los procesos.

También, la dualidad de criterios al instruir líneas de acción, poco manejo por parte del director de leyes y reglamentos de la normativa vigente que rigen la materia; también podría estar incidiendo en esta situación el desconocimiento de funciones, atribuciones, competencias de este personal, unido posiblemente a las limitantes que existen en el sector para aplicar mecanismos de control y sanciones.

Las situaciones descritas anteriormente, conforman parte de las responsabilidades del cuerpo directivo en su día a día, donde debe brindar orientaciones y soluciones a requerimientos específicos, tales como: levantamientos de actas, vaciado de información en formatos especiales, revisión y acatamiento de la normativa que rige la educación bolivariana, procedimientos sancionatorios, mediación, entre otros; son parte de las actividades administrativas que se han de cumplir en todo plantel educativo.

Cabe destacar que la falta de seguimiento por parte del personal administrativo a los docentes que incurren en el cumplimiento de los recaudos y las continuas modificaciones en cortos lapsos de tiempo repercute en el 
OAI-PMH: http://www.indteca.com/ojs/index.php/Revista_Scientific/oai

Artículo Original / Original Article

desarrollo de las actividades académicas del estudiantado y por consiguiente en su rendimiento académico.

El presente artículo tiene como propósito analizar la gestión de la administración escolar en el desarrollo de las actividades académicas en tiempos de pandemia en el Liceo Técnico Amelia Courbis, provincia de Talca. Chile; induciendo que en la praxis educativa el proceso administrativo insta a desarrollar la planificación, organización, ejecución y control de las diferentes actividades que se llevan a cabo dentro de los entornos educativos.

\section{Desarrollo}

\subsection{Gestión de la Administración Escolar}

El quehacer educativo como proceso se fundamenta en una visión holística donde sus deberes y derechos se sustentan en una sociedad cambiante como la actual, donde el proceso debe ser más exigente en cuanto a las formas de convivencia, las maneras de resolver conflictos, la comunicación, las relaciones interpersonales y, para ello se debe definir las funciones que cumple cada uno de ellos, en este caso, el de los directivos, docentes, padres y representantes.

Por ello, Pacheco-Granados, Robles-Algarín y Ospino-Castro (2018): exponen que la gestión de la administración escolar representa el conjunto de acciones que desarrolla el directivo en función de elaborar la planificación, organización, control y seguimiento a las actividades académicas, recursos, materiales, definición de gastos, entre otros. De esta manera, el quehacer administrativo parte con la planificación, espacio donde se bordan las metas a lograr.

Parafraseando a García, Juárez y Salgado (2018): la administración escolar es la actividad de llevar con dedicación y talento en alcanzar las metas, objetivos y estrategias que utilizan los directivos o gerentes en la consecución de la calidad, efectividad y efectividad de la educación a través de las 
relaciones interpersonales entre los integrantes de su equipo, comunicación, tomada de decisiones, liderazgo y satisfacción laboral.

La gestión de la administración escolar, señalando a Gallegos (2017): es el proceso mediante el cual el equipo directivo establece las actividades planificadas de acuerdo a los objetivos propuestos en la institución tomando en cuenta la necesidades e intereses, transformaciones, acciones para el presente y proyecciones hacia el futuro en función a los logros que se quieren alcanzar. El director es el responsable directo de la administración.

Por esto el ejercicio de la acción directiva tiene un alcance social requiere de directores con una formación renovada permanentemente para poder dirigir eficientemente las instituciones educativas. El director no es un funcionario de simples condiciones, para ello, debe estar capacitado, ser un líder nato, principal responsable de la gerencia del proceso educativo del plantel, como gerente educacional cumple con los procesos administrativos que se realizan en cualquiera de los niveles educativos de la estructura formal del sistema.

\subsection{Actividades académicas}

Las actividades académicas imbricadas en los resultados que se obtienen en la calidad de la educación en función de mejorar los procesos administrativos y académicos en una institución escolar. En este sentido, Hamui-Sutton, Lavalle-Montalvo, Díaz-Villanueva, Gómez-Lamont, CarrascoRojas y Vilar-Puig (2013): exponen que "las actividades académicas teóricas son aquellas donde los roles de maestro-alumno son muy claros, uno está en la disposición de enseñar y el otro a aprender" (pág. 26); significa que el profesor es el responsable de guiar, orientar y asesorar a los estudiantes en las acciones inherentes al proceso enseñanza-aprendizaje.

En efecto, García, Fonseca y Concha (2015): presentan que las actividades ejercidas dentro del contexto educativo, exigen actitudes y 
OAI-PMH: http://www.indteca.com/ojs/index.php/Revista_Scientific/oai

Artículo Original / Original Article

habilidades que se conjugan con el trabajo colaborativo intercambiando experiencias $\mathrm{y}$, por ende, saberes promoviendo las capacidades propias del desempeño profesional como educadores a fin lograr aprendizajes significativos en los alumnos.

También, representan un sistema orientado a la asesoría planificada en la cual permite realizar un seguimiento, contextualización, dinamismo y control, en las actividades administrativas y académicas orientadas a mejorar la calidad de educativa y por ende el rendimiento de los estudiantes. Además, constituyen, transformaciones logrando la visión holística del ser humano, como entre imbuido dentro de una sociedad en desarrollo sostenido.

\subsection{Pandemia}

La génesis y revolución relacionada con la Pandemia COVID-19, que trajo consigo transformaciones, cambios de conducta, estilos de vida diferentes en cada uno de los individuos y, en especial en el funcionamiento de las universidades. Como dice Espada, Orgilés, Piqueras y Morales (2020): el afrontamiento de esta pandemia está colocando a prueba la capacidad de acomodo de manera propia y colectiva en la vida de las personas; a la vez las cuantiosas transformaciones por esta crisis y su impacto social en la promoción de comportamientos ante estos nuevos escenarios.

Por lo tanto, la gestión de la administración escolar, en tiempos de crisis o pandemia, vino a modificar abruptamente todos los escenarios a los cuales se estaba acostumbrado. Algunas instituciones, especialmente en el escenario educativo, ha cobrado mayor relevancia e impacto, razón por la cual se han tenido que valerse de muchas acciones, estrategias, técnicas y herramientas tecnológicas para continuar con las funciones de docencia, investigación y extensión.

Dichas acciones se llevan a cabo para proteger las remuneraciones de sus profesores, la continuidad en la formación de los estudiantes, la 
investigación y extensión de todas sus actividades, interpretando a GarcíaMuñoz, Pérez y Navarrete (2020): plantean que es el momento de actuar rápido y fortalecer la sostenibilidad y seguir adelante. Además, se deben asumir los cambios o transformaciones en función de mejorar actitudes hacia el bienestar de los integrantes de los establecimientos escolares a través del contacto abierto, reflexible y permanente.

Por lo tanto, en Chile la interrupción de las clases presenciales en todo el territorio chileno, en la cual aceleradamente se implementó la educación a distancia o remota. Representó un reto para los directores ya que se han visto en la necesidad de girar sus actividades desde otra visión. Es decir, los gerentes se han tenido que valerse de diferentes herramientas con el propósito de continuar con la gestión administrativa escolar.

\section{Método}

El presente artículo se inserta en el enfoque cuantitativo que conforme a Palella y Martins (2012): "la investigación cuantitativa requiere el uso de instrumentos de medición y comparación, que proporcionan datos cuyo estudio necesita la aplicación de modelos matemáticos y estadísticos" (pág. 46); para una mejor explicación de los hechos se recolectan los datos mediante la aplicación de instrumentos que previamente fueron elaborados y diseñados con el fin de recabar información mediante técnicas estadísticas.

Respecto el diseño no experimental, siguiendo a Hernández y Mendoza (2018): son estudios que no requieren la manipulación de variables y los sujetos participantes son examinados directamente de la realidad, con un alcance es descriptivo. En este sentido, la población estuvo constituida por veinticinco (25) directivos y docentes del Liceo Técnico Amelia Courbis, ubicado la provincia de Talca, Región del Maule, Chile.

Se utilizó la técnica de muestreo del censo de población, se tomó como muestra la totalidad de la población, esta fue no probabilística e intencional 
OAI-PMH: http://www.indteca.com/ojs/index.php/Revista_Scientific/oai

Artículo Original / Original Article

porque dependió de la decisión de los investigadores, los objetivos planteados y la intención del análisis de las estrategias de acompañamiento. Asimismo, se pretendió contribuir con conocimiento científico valido para los sujetos participantes.

El instrumento empleado fue un cuestionario con escalamiento tipo Likert con cinco alternativas de respuesta: Siempre, Casi Siempre, A Veces, Casi Nunca y Nunca, en conformidad con Hernández, Fernández y Baptista (2014): con un total de ocho (08) ítems en la cual las ponderaciones altas muestran una actitud más positiva a las respuestas emitidas en las categorías.

La validación del instrumento se formalizó a través de los análisis de contenido por medio de tres expertos en metodología, estadística y supervisores educativos, quienes concordaron que el cuestionario se encontraba a acorde al estudio de las variables. Seguidamente, se aplicó una prueba piloto a 20 docentes, que no pertenecían a la muestra de estudio y tenían las mismas características. De esta manera, se usó el coeficiente de alfa Cronbach, obteniendo un valor de 0,93 considerándose confiable.

Del mismo modo, el análisis de los datos fue realizado con la utilización del software estadístico para las ciencias sociales, IBM SPSS Statistics, por sus siglas en inglés, en su versión 20; representándose por medio de tablas, donde se presentó las frecuencias absolutas y los porcentajes de las respuestas emitidas por los sujetos participantes a las dimensiones de estrategias de acompañamiento y estudios a distancia, esto unido a la utilización de Excel, ya que los datos se organizaron y filtraron para su posterior análisis.

\section{Resultados}

Una vez finalizada la etapa de procesamiento de la información, se recurrió a analizar los datos recabados haciendo uso del análisis estadístico, el cual, está orientado por el diseño de investigación. En el caso que aquí 
ocupa se presentaran los resultados que emanan de la aplicación del instrumento que fue aplicado al personal docente, coordinadores y directivo adscrito al liceo antes mencionado.

Una vez obtenidos los datos y clasificados estos, se procedió a efectuar la tabulación de los mismos a través de la construcción de tablas de frecuencia, porcentajes específicos y su representación mediante gráficos de barras que representan las categorías Siempre, Casi Siempre, A Veces, Casi Nunca y Nunca, suministrando de tal forma los cimientos requeridos para cristalizar el proceso de análisis de los datos como se describen a continuación.

Tabla 1. Distribución de la frecuencia de la variable Procesos Administrativos en su dimensión Funciones Administrativas para los indicadores Planificación, Dirección, Organización y Control.

\begin{tabular}{|c|c|c|c|c|c|c|c|c|c|c|c|c|}
\hline \multirow{3}{*}{ ÍTEMS } & \multicolumn{12}{|c|}{ Alternativas de Respuesta } \\
\hline & \multicolumn{2}{|c|}{ Siempre } & \multicolumn{2}{|c|}{$\begin{array}{c}\text { Casi } \\
\text { Siempre }\end{array}$} & \multicolumn{2}{|c|}{$\begin{array}{c}\text { A } \\
\text { Veces }\end{array}$} & \multicolumn{2}{|c|}{$\begin{array}{c}\text { Casi } \\
\text { Nunca }\end{array}$} & \multicolumn{2}{|c|}{ Nunca } & \multicolumn{2}{|c|}{ Totales } \\
\hline & $\mathbf{F}$ & $\%$ & $\mathbf{F}$ & $\%$ & $\mathbf{F}$ & $\%$ & $\mathbf{F}$ & $\%$ & $\mathbf{F}$ & $\%$ & $\mathbf{F}$ & $\%$ \\
\hline $\begin{array}{l}\text { 1. En la institución se } \\
\text { establece una } \\
\text { planificación articulada } \\
\text { con todos los niveles del } \\
\text { sistema educativo. }\end{array}$ & 13 & 52 & 11 & 44 & 1 & 4 & 0 & 0 & 0 & 0 & 25 & 100 \\
\hline $\begin{array}{l}\text { 2. El gerente en su } \\
\text { administración delega } \\
\text { funciones para cumplir } \\
\text { con los objetivos de la } \\
\text { institución. }\end{array}$ & 11 & 44 & 11 & 44 & 2 & 8 & 1 & 4 & 0 & 0 & 25 & 100 \\
\hline $\begin{array}{c}\text { 3. El gerente diseña la } \\
\text { estructura organizativa } \\
\text { de la institución } \\
\text { tomando en cuenta las } \\
\text { capacidades del } \\
\text { personal. }\end{array}$ & 0 & 0 & 4 & 16 & 4 & 16 & 17 & 68 & 0 & 0 & 25 & 100 \\
\hline $\begin{array}{l}\text { 4. El gerente organiza la } \\
\text { estructura de la escuela } \\
\text { asociada a los objetivos } \\
\text { establecidos. }\end{array}$ & 5 & 20 & 17 & 68 & 2 & 8 & 1 & 4 & 0 & 0 & 25 & 100 \\
\hline
\end{tabular}


OAI-PMH: http://www.indteca.com/ojs/index.php/Revista_Scientific/oai

Artículo Original / Original Article

\begin{tabular}{|c|c|c|c|c|c|c|c|c|c|c|c|c|}
\hline $\begin{array}{c}5 . \text { El personal que } \\
\text { administra la institución } \\
\text { vigila que se cumplan } \\
\text { todas las órdenes } \\
\text { emitidas. }\end{array}$ & 1 & 4 & 3 & 12 & 8 & 32 & 13 & 52 & 0 & 0 & 25 & 100 \\
\hline
\end{tabular}

Fuente: Las Autoras (2020).

En la tabla 1, que contiene la información sobre los datos obtenidos en el ítem 1 , se observa que el $52 \%$ del personal encuestado manifiesta que en la institución "Siempre" se establece una planificación articulada con todos los niveles del sistema educativo, el $44 \%$ en la alternativa "Casi Siempre", el $4 \%$ manifestaron que "A Veces" se hace y el resto de encuestados señalaron en las opciones "Casi Nunca" y "Nunca". De acuerdo a estos resultados se pudo inferir que en la institución se lleva a cabo el proceso administrativo de planificación articulado con todos los niveles del sistema.

En este sentido, se considera cierta afinidad con las afirmaciones de Ander-Egg (2015): cuando expresa que "la planificación educativa para ser eficaz, debe ser articulada y sistemática en su práctica, en todos los niveles del sistema" (pág. 54). Significa que la planificación debe estar articulado o engranada con la institución escolar y el Ministerio de educación, razón por la cual se busca el logro de metas propuestas mancomunadas.

Seguidamente, se encuentra el ítem 2, el $44 \%$ en las alternativas de "Siempre" y 44\% de "Casi Siempre" afirman que el gerente en su administración delega funciones para cumplir con los objetivos de la institución, en contra parte con un $8 \%$ manifiesta que "A Veces" y por último el cuatro $4 \%$ del personal encuestado considero que "Casi Nunca", pudiéndose constatar que en su gestión el director lleva a cabo el proceso administrativo de dirección al delegar funciones a su personal para cumplir con los objetivos de la institución educativa.

En este marco, Cruzata y Rodríguez (2016a): reseñan que el gerente educativo debe ejercer la dirección de su institución poniendo en marcha los 
proyectos educativos establecidos y a su vez estableciendo estrategias que se rijan por las establecidas o en las políticas educativas vigentes, acompañado por el grupo de docentes a su cargo, permitiéndoles la participación y delegándole funciones.

Para continuar se encuentra el ítem 3 referido a la Organización como proceso administrativo para ello se le preguntó a los encuestados si el gerente diseña la estructura organizativa de la institución tomando en cuenta las capacidades del personal, encontrándose el 68\% manifestó que "Casi Nunca" lo hace, seguidamente se observa una tendencia del $16 \%$ opina entre las alternativas "Casi Siempre" y 16\% consideran "A Veces" logra diseñar la estructura organizativa de la institución tomando en cuenta las capacidades del personal, y por último los restantes se inclinaron por las opciones "Nunca" y "Siempre", lo que viene a constituir una desproporción en la población objeto de estudio ya que una mayoría de los encuestados manifestó que el gerente Casi Nunca toma en cuenta las capacidades del personal al momento de diseñar la estructura organizativa de la institución.

En este aspecto, se observa que el gerente encargado de diseñar la estructura organizativa de la institución no cumple con el proceso administrativo de organización que, de acuerdo a lo indicado por Abad (2018): establece las acciones diseñando las motivaciones propias del ser humano.

El ítem 4, conexo con la organización como proceso administrativo donde se le pregunto al personal encuestado si el gerente organiza la estructura de la escuela asociada a los objetivos establecidos, para lo que el $68 \%$ respondió que "Casi Siempre" lo hace, mientras que un 20\% manifestó que "Siempre", el 8\% "A Veces" y un 4\% "Casi Nunca" el gerente organiza la estructura de la escuela asociada a los objetivos establecidos, mientras que ninguna persona se inclinó por la opción.

En este sentido, López, García y Martínez (2019): manifiestan que en toda institución educativa existe un nivel jerárquico consustanciado en las 
metas consolidadas en la normativa que rige los procesos administrativos, determinando de esta manera las tareas, funciones e interrelaciones entre los diversos cargos que se plantean en la institución educativa.

Asimismo, se encuentra el ítem 5 relacionado con el control, uno de los procesos administrativos más importantes dentro de la administración de cualquier institución, para lo cual se le pregunto al personal encuestado si el personal que administra la institución vigila que se cumplan todas las órdenes emitidas, para lo el $52 \%$ contesto que "Casi Nunca" se vigila que se cumplan todas las órdenes emitidas, el 32\% "A Veces", un 12\% manifestó que "Casi Siempre" y $4 \%$ "Siempre".

Con estos resultados se observa desarticulación con las ideas de Cruzata y Rodríguez (2016b): quienes plantean que controlar el funcionamiento de los planteles educativos implica analizar las causas de los resultados obtenidos, con el propósito de corregir las desviaciones y mantener o mejorar los logros. Lo que hace evidente que, si no se analizan los resultados obtenidos al término del lapso de planificación, no podrán corregirse las desviaciones.

Tabla 2. Distribución de la frecuencia de la variable Gerencia Educativa en su dimensión Rol del Gerente Educativo en el Proceso Administrativo para el indicador Características del director.

\begin{tabular}{|c|c|c|c|c|c|c|c|c|c|c|c|c|}
\hline \multirow{3}{*}{ ÍTEMS } & \multicolumn{12}{|c|}{ Alternativas de Respuesta } \\
\hline & \multicolumn{2}{|c|}{ Siempre } & \multicolumn{2}{|c|}{$\begin{array}{c}\text { Casi } \\
\text { Siempre }\end{array}$} & \multicolumn{2}{|c|}{$\begin{array}{c}A \\
\text { Veces }\end{array}$} & \multicolumn{2}{|c|}{$\begin{array}{c}\text { Casi } \\
\text { Nunca }\end{array}$} & \multicolumn{2}{|c|}{ Nunca } & \multicolumn{2}{|c|}{ Totales } \\
\hline & $\mathbf{F}$ & $\%$ & $\mathbf{F}$ & $\%$ & $\mathbf{F}$ & $\%$ & $\mathbf{F}$ & $\%$ & $\mathbf{F}$ & $\%$ & $\mathbf{F}$ & $\%$ \\
\hline $\begin{array}{l}\text { 6. Considera que el } \\
\text { director de la escuela } \\
\text { mantiene una formación } \\
\text { renovada permanente } \\
\text { para dirigir eficientemente } \\
\text { la educación remota en } \\
\text { tiempo de pandemia }\end{array}$ & 0 & 0 & 3 & 12 & 7 & 28 & 12 & 48 & 3 & 12 & 25 & 100 \\
\hline $\begin{array}{l}\text { 7. A su juicio el director } \\
\text { logra la buena marcha de } \\
\text { los procesos } \\
\text { administrativos en tiempos } \\
\text { de pandemia. }\end{array}$ & 1 & 4 & 4 & 16 & 6 & 24 & 12 & 48 & 2 & 8 & 25 & 100 \\
\hline
\end{tabular}




\begin{tabular}{|c|c|c|c|c|c|c|c|c|c|c|c|c|}
\hline $\begin{array}{c}\text { 8. Considera que el } \\
\text { director aplica estrategias } \\
\text { administrativas que son } \\
\text { efectivas en la educación } \\
\text { remota. }\end{array}$ & 1 & 4 & 2 & 8 & 4 & 16 & 10 & 40 & 8 & 32 & 25 & 100 \\
\hline
\end{tabular}

Fuente: Autores (2020).

Para la tabla 2, se pudo determinar para el ítem 6 el $48 \%$ respondió en atención al criterio "Casi Nunca" el director de la escuela mantiene una formación renovada permanente para dirigir eficientemente la educación remota en tiempos de pandemia, el 28\% "A Veces"; el 12\% se ubicó en las alternativas "Casi Siempre" y $12 \%$ en "Nunca" y los restantes en alternativa "Siempre". Se observa la falta de manejo del directivo de acercar efectivamente la educación en tiempos de pandemia.

En el ítem 7, el personal encuestado sobre, a su juicio el director logra la buena marcha de los procesos administrativos en tiempos de pandemia, estos contestaron un 48\% "Casi Nunca", 24\% "A Veces", el 16\% "Casi Siempre", el $8 \%$ Nunca y $4 \%$ "Siempre"; infiriendo que el equipo directivo carece de una buena práctica en los procesos administrativos en tiempos de crisis y proceso transitorios.

Para el ítem 8, se pudo determinar que, para el criterio, considera que el director aplica estrategias administrativas que son efectivas en la educación remota, el 40\% respondió que "Casi Nunca", el 32\% "Nunca", el 16\% "A Veces", el $8 \%$ "Casi Siempre" y un 4\% "Siempre". Los resultados obtenidos evidencian algunas debilidades en el aspecto relacionado, por cuanto el director como gerente es la máxima autoridad y por ende debe mejorar los procesos en tiempos de pandemia.

Con estos resultados se observa desarticulación con las ideas de Jiménez (2016), citado por Herrera y Tobón (2017): consideran que el director debe estar preparado adecuadamente para el ejercicio de su cargo, cuyo rol se conduzca hacia la determinación y el logro de los objetivos y metas que se 
aspiren lograr.

\section{Discusión}

Después de procesados estadísticamente los resultados y discutidos a la luz de las teorías consultadas, se pudo diagnosticar que dentro de los procesos administrativos que lleva a cabo la gerencia educativa del liceo está la planificación articulada con todos los niveles del sistema. Sin embargo, el Control presenta gran problemática en su aplicación debido a que no se está cumpliendo con el proceso rigurosamente poniendo en peligro el logro de los objetivos planteados en la planificación, pues, el ejecutivo no está verificando si los hechos van de acuerdo con los objetivos.

De igual manera se encuentra la organización donde no se toma en cuenta las capacidades del personal al diseñar la estructura organizativa de la institución, provocando desmotivación en el personal, dado a que se encuentran inconformes con las funciones que desempeñan dentro de la institución escolar. Aunado a esta situación, se encuentra el caso de que el personal que se encuentra a cargo de la administración de la escuela no tiene el conocimiento necesario para responder ante entes superiores debido al poco manejo de leyes y reglamentos, así como el desconocimiento de funciones, atribuciones y competencias que obedecen a este personal

De acuerdo a la opinión emitida por los encuestados se determina que los mismos perciben que el director como gerente de la institución no posee la preparación necesaria para llevar a cabo la administración en la educación remota en tiempos de pandemia, debido a que no reúne las competencias para el cargo, demostrando esto al no llevar la buena marcha de los procesos administrativo ya que no aplica estrategias administrativas efectivas para el cargo,

Al igual el hecho que no se brinda el proceso de seguimiento y control, tan necesarios para mejorar la praxis pedagógica del docente, para ello es 
importante resaltar la necesidad que presenta el equipo directivo de formarse sobre todo en la aplicación de procedimientos educativos, para lo cual requiere poseer conocimiento sobre el aspecto legal que rige el sistema educativo venezolano, primordialmente en lo referente a los procedimientos administrativos a realizar dentro de la institución.

Es importante que los directivos se pongan a tono con los nuevos cambios y transformaciones, razón por la cual la educación remota, que fue implementada bruscamente producto de la pandemia COVID-19, vino para quedarse, por lo tanto, el equipo directivo debe organizar, planificar, direccionar y controlar desde los escenarios remotos, de la mano con los docentes y demás miembros en la consolidación de estrategias educativa que impliquen mejorar la gestión administrativa escolar.

\section{Referencias}

Abad, S. (2018). La gestión educativa y el liderazgo pedagógico en la Escuela Superior de Música Pública “José María Valle Riestra” de Piura, 2018. Tesis de Grado. Piura, Perú: Universidad César Vallejo. Recuperado de: https://hdl.handle.net/20.500.12692/28859

Ander-Egg, E. (2015). Introducción a la planificación estratégica. Buenos Aires, Argentina: Editorial Humanitas, S.L.

Chávez, C. (2017). Estrés, Salud y Psicopatología Laborales: Síndrome de Burnout en Profesionales de la Educación Superior en Ecuador. Tesis Doctoral. España: Universidad de Extremadura. Recuperado de: http://hdl.handle.net/10662/6469

Cruzata, A., \& Rodríguez, I. (2016a,b). La Gestión en las Instituciones Educativas: Enfoques, Modelos y Posiciones Teóricas y Prácticas. Revista Gobierno y Gestión Pública, 3(1), 28-37, e-ISSN: 2414-4991. Recuperado de:

http://www.revistagobiernoydegestionpublica.com/index.php/RGGP/arti 
cle/view/31

Espada, J., Orgilés, M., Piqueras, J., \& Morales, A. (2020). Las Buenas Prácticas en la Atención Psicológica Infanto-juvenil ante el COVID19. Revista de Clínica y Salud, 31(2), 109-113, e-ISSN: 1130-5274. Recuperado de: https://doi.org/10.5093/clysa2020a14

Fierro-Evans, C., \& Carbajal-Padilla, P. (2019). Convivencia escolar: Una revisión del concepto. Psicoperspectivas. Individuo y Sociedad, 18(1), 1-19, e-ISSN: 0718-6924. Recuperado de:

http://dx.doi.org/10.5027/psicoperspectivas-vol18-issue1-fulltext-1486

Gallegos, Y. (2017). Gestión escolar de las directoras de instituciones educativas del nivel inicial de la ciudad de puno en el año 2016. Tesis. Puno, Perú: Universidad Nacional del Altiplano. Recuperado de: http://repositorio.unap.edu.pe/handle/UNAP/6579

García, F., Fonseca, G., \& Concha, L. (2015). Aprendizaje y rendimiento académico en educación superior: Un estudio comparado. Revista Electrónica "Actualidades Investigativas en Educación", 15(3), 1-26, eISSN: 1409-4703. Recuperado de:

https://www.redalyc.org/articulo.oa?id=44741347019

García, F., Juárez, S., \& Salgado, L. (2018). Gestión escolar y calidad educativa. Revista Cubana Educación. Revista Cubana Educación Superior, 37(2), 206-216, e-ISSN: 2518-2730. Recuperado de: http://www.rces.uh.cu/index.php/RCES/article/view/223

García-Muñoz, C., Pérez, B., \& Navarrete, M. (2020). Las empresas antel el COVID-19. Revista de Investigación en Gestión Industrial, Seguridad y Salud en el Trabajo - GISST, 2(2), 85-101, e-ISSN: 2711-2934. Recuperado de:

https://www.editorialeidec.com/revista/index.php/GISST/article/view/83 $\underline{164}$

Hamui-Sutton, A., Lavalle-Montalvo, C., Díaz-Villanueva, A., Gómez-Lamont, 
D., Carrasco-Rojas, J., \& Vilar-Puig, P. (2013). Las actividades académicas en contextos clínicos por especialidades: percepción de los estudiantes. Investigación en Educación Médica, 2(5), 25-36, eISSN: 2007-5057. Recuperado de:

http://www.scielo.org.mx/scielo.php?script=sci arttext\&pid=S2007$\underline{50572013000100005}$

Hernández, R., \& Mendoza, C. (2018). Metodología de la investigación: Las rutas cuantitativa, cualitativa y mixta. ISBN: 978-1-4562-6096-5. México: Editorial McGraw-Hill Education.

Hernández, R., Fernández, C., \& Baptista, M. (2014). Metodología de la Investigación. Sexta edición, ISBN: 978-1-4562-2396-0. México: McGraw-Hill / Interamericana Editores, S.A. de C.V.

Herrera, S., \& Tobón, S. (2017). El director escolar desde el enfoque socioformativo. estudio documental mediante la cartografía conceptual. Revista de Pedagogía, 38(102), 164-194, e-ISSN: 07989792. Recuperado de:

https://www.redalyc.org/articulo.oa?id=65952814009 López, E., García, L., \& Martínez, J. (2019). La gestión directiva como potenciadora de la mejora del clima organizacional y la convivencia en las instituciones de educación media superior. Ride: Revista Iberoamericana para la Investigación y el Desarrollo Educativo, 9(18), 792-812, e-ISSN: 2007-7467. Recuperado de: https://doi.org/10.23913/ride.v9i18.471

Miranda, S. (2016). La gestión directiva: un concepto construido desde las comprensiones de los directivos docentes de las escuelas públicas bogotanas. Ride: Revista Iberoamericana para la Investigación y el Desarrollo Educativo, 7(13), 562-589, e-ISSN: 20077467. Recuperado de:

http://www.scielo.org.mx/scielo.php?script=sci arttext\&pid=S2007- 
OAI-PMH: http://www.indteca.com/ojs/index.php/Revista_Scientific/oai

Artículo Original / Original Article

\section{$\underline{74672016000200562}$}

Muñoz, D., \& Villa, E. (2017). Paulo Freire en la educación popular latinoamericana: el porqué y el para qué de estarse formando como pueblo político. Kavilando, 9(1), 276-286, e-ISSN: 2027-2391. Recuperado de:

https://dialnet.unirioja.es/servlet/articulo?codigo $=6110066$

Pacheco-Granados, R., Robles-Algarín, C., \& Ospino-Castro, A. (2018). Análisis de la Gestión Administrativa en las Instituciones Educativas de los Niveles de Básica y Media en las Zonas Rurales de Santa Marta, Colombia. Información Tecnológica, 29(5), 259-266, e-ISSN: 0718-0764. Recuperado de: http://dx.doi.org/10.4067/S0718$\underline{07642018000500259}$

Palella, S., \& Martins, F. (2012). Metodología de la Investigación Cuantitativa. 1ra reimpresión, ISBN: 980-273-445-4. Caracas, Venezuela: Fondo Editorial de la Universidad Pedagógica Experimental Libertador - FEDUPEL. 


\section{Amely Dolibeth Vivas Escalante}

e-mail: amelydvivase@gmail.com

Nacida en San Cristóbal, Venezuela, el 3 de enero del año

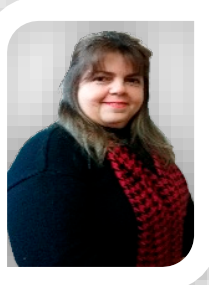
1970. Docente Académico-Investigador con PostDoctorado en Estudios Libres; Doctora en Ciencias de la Educación, titulada en la Universidad Fermín Toro (UFT); Magíster en Planificación Educativa por la Universidad Pedagógica Experimental Libertador (UPEL); Especialista en Evaluación Educacional en la UPEL; Licenciada en Educación Mención Matemáticas, en la Universidad de Los Andes (ULA); Experiencia como profesor Universitario a nivel de pregrado y postgrado, tutora, jurado y asesora de trabajos de investigación y tesis doctorales. 
OAI-PMH: http://www.indteca.com/ojs/index.php/Revista_Scientific/oai

\section{Artículo Original / Original Article}

\section{Marlenis Marisol Martínez Fuentes}

e-mail: marlenism3@gmail.com

Nacida en el estado Apure, Venezuela, el 1 de septiembre

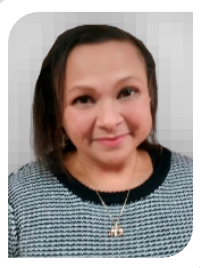
del año 1969. Actualmente me desempeño como docente académico investigador en la Universidad Miguel de Cervantes (UMC), en Santiago de Chile; mis estudios académicos son Post-Doctor en Gestión del Conocimiento por la Universidad Pedagógica Experimental Libertador (UPEL); Doctor en Ciencias de la Educación en la Universidad Fermín Toro (UFT); Magíster en Educación, Mención Gerencia Educacional; y Licenciada en Educación Integral, Mención Castellano y Literatura y Ciencias Naturales de la Universidad Experimental de los Llanos Occidentales Ezequiel Zamora (UNELLEZ), de Barinas, Venezuela; he participado en conferencias nacionales e internacionales relacionadas con educación, como ponente y participante; cuento con experiencia como profesor Universitario durante 17 años a nivel de pregrado y postgrado, tutora, jurado y asesora de trabajos de investigación y tesis doctorales. 
OAI-PMH: http://www.indteca.com/ojs/index.php/Revista_Scientific/oai

Artículo Original / Original Article

e-mail: dorisolis@hotmail.com

Nacida en Venezuela, el 1 de febrero del año 1970.

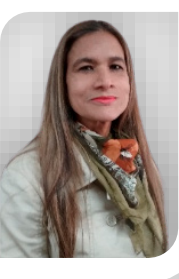

Docente Académico-Investigador de la Universidad Miguel de Cervantes (UMC), Santiago de Chile; con Magister en Gerencia y Liderazgo en Educación; Licenciada en Educación Integral, mención Castellano y Literatura y Ciencias Sociales; Académica de la Universidad Miguel de Cervantes (UMC); Ponente en Conferencias relacionadas con el área de Logística, Matemáticas, Investigación y aspectos relacionados; y Relatora en cursos de clima organizacional y organización empresarial.

El contenido de este manuscrito se difunde bajo una Licencia de Creative Commons ReconocimientoNoComercial-Compartirlgual 4.0 Internacional 